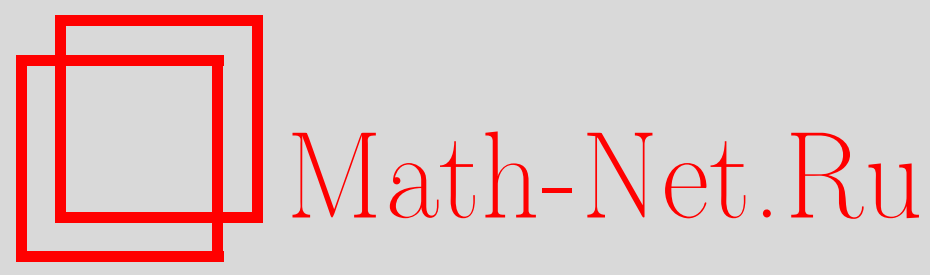

В. А. Ватутин, Е. Е. Дьяконова, Разложимые ветвящиеся процессы с двумя типами частиц, Дискрет. матем., 2018, том 30, выпуск 1, 3-18

DOI: https://doi.org/10.4213/dm1475

Использование Общероссийского математического портала Math-Net.Ru подразумевает, что вы прочитали и согласны с пользовательским соглашением http://www.mathnet.ru/rus/agreement

Параметры загрузки:

IP : 34.239 .49 .27

26 апреля 2023 г., 13:01:42 


\title{
Разложимые ветвящиеся процессы с двумя типами частиц
}

(ㄱ 2018 г. В. А. Ватутин*, Е. Е. Дьяконова**

\begin{abstract}
Рассматривается критический разложимый ветвящийся процесс Гальтона-Ватсона с двумя типами частиц, в котором частицы первого типа производят в конце жизни как потомков первого типа, так и потомков второго типа, а частицы второго типа порождают в момент гибели лишь потомков своего типа.

В предположении, что распределение числа потомков у частицы каждого типа может иметь бесконечную дисперсию, найдена асимптотика хвоста распределения случайной величины $\Xi_{2}-$ совокупного числа частиц второго типа, появившихся в процессе до момента его вырождения. Доказаны предельные теоремы, описывающие (при $N \rightarrow \infty$ ) условное распределение числа частиц первого типа в различных поколениях, как при условии $\Xi_{2}=N$, так и при условии $\Xi_{2}>N$.
\end{abstract}

Исследование выполнено за счет гранта Российского научного фонда (проект 14-50-00005).

Ключевые слова: разложимый ветвящийся процесс, совокупный размер популяции, предельная теорема

\section{1. Введение}

Рассмотрим ветвящийся процесс Гальтона-Ватсона с двумя типами частиц, в котором частицы первого типа производят в конце жизни как потомков первого типа, так и потомков второго типа, а частицы второго типа порождают в момент гибели лишь потомков своего типа.

Обозначим $Z_{1}(n)$ и $Z_{2}(n)$ числа частиц, соответственно, первого и второго типов в $n$-м поколении рассматриваемого ветвящегося процесса, и введем вектор $\mathbf{Z}(n)=\left(Z_{1}(n), Z_{2}(n)\right), n \geqslant 0$. В дальнейшем (если не оговорено иное) мы будем предполагать, что $\mathbf{Z}(0)=\mathbf{e}_{1}:=(1,0)$.

Процесс $\mathbf{Z}(n)$ принадлежит классу так называемых разложимых ветвящихся процессов, исследованием которых занимались многие авторы. Причину интереса к

*Место работы: Математический институт им. В.А. Стеклова Российской академии наук, e-mail: vatutin@mi.ras.ru

${ }^{* *}$ Место работы: Математический институт им. В.А. Стеклова Российской академии наук, e-mail: elena@mi.ras.ru 
изучению таких процессов легко объяснить: при анализе их свойств удалось обнаружить ряд новых интересных явлений, не имеющих аналогов для неразложимых ветвящихся процессов. Это касается как разнообразия в асимптотическом поведении вероятностей невырождения критических и докритических процессов ([13], [14]), так и появления новых классов условных предельных теорем для критических процессов ([9], [11], [12], [15], [23]).

В последнее время был обнаружен целый ряд принципиально новых свойств, присущих только разложимым критическим процессам. Например, в [4], [5] был описан эффект фазового перехода для редуцированных разложимых критических процессов Гальтона-Ватсона с конечными дисперсиями чисел потомков всех типов при условии невырождения этих процессов к далекому моменту $N$. Эти результаты были дополнены в статье [21], где при условии невырождения разложимых процессов с двумя типами частиц к далекому моменту $N$ было изучено асимптотическое поведение распределения числа частиц редуцированных процессов в ситуации, когда дисперсии чисел потомков частиц могут быть бесконечными.

В работах [7], [8] наличие фазовых переходов было обнаружено для разложимых критических процессов Гальтона-Ватсона с конечными дисперсиями чисел потомков всех типов в схеме серий когда моменты вырождения таких процессов фиксированы, но стремятся к бесконечности.

Вместо условия невырождения процесса к данному моменту $N$ или условия вырождения процесса точно в момент $N$ в ряде статей рассматривались распределения разложимых процессов при условии, что фиксированы значения случайных величин

$$
\Xi_{i}(n):=\sum_{j=0}^{n} Z_{i}(j), \quad \Xi_{i}:=\sum_{j=0}^{\infty} Z_{i}(j)
$$

- общего числа частиц типа $i$, появившихся в процессе за $n$ поколений, и совокупного числа частиц типа $i$, появившихся в процессе до момента вырождения, соответственно.

Так, в $[1,2]$ изучались разложимые ветвящиеся процессы с двумя типами частиц в ситуации, когда дисперсии чисел потомков частиц конечны. В указанных статьях были исследованы распределения процессов $\left\{Z_{1}(t \sqrt{N}), 0 \leqslant t<\infty\right\}$ и $\left\{\Xi_{2}(t N), 0 \leqslant t<\infty\right\}$ при условии $\Xi_{1}=N \rightarrow \infty$, а также найдено предельное распределение случайной величины $\Xi_{1}$ при условии $\Xi_{2}>N \rightarrow \infty$. В статье [3] (снова в случае конечности дисперсий чисел потомков частиц каждого типа) была установлена функциональная предельная теорема для процесса $\left\{Z_{1}(t \sqrt[4]{N}), 0 \leqslant t<\infty\right\}$ при условии $\Xi_{2}>N \rightarrow \infty$.

В [6] от предположения о конечности дисперсий чисел потомков частиц удалось отказаться, и была доказана функциональная предельная теорема для процесса $\left\{\Xi_{2}(t N), 0 \leqslant t<\infty\right\}$ при условии $\Xi_{1}=N \rightarrow \infty$.

В данной работе, дополняющей [1], [2] и [6], мы исследуем распределение числа частиц первого типа в разложимом ветвящемся процессе с двумя типами частиц и с (возможно) бесконечными дисперсиями чисел потомков при различных условиях, накладываемых на величину $\Xi_{2}$.

Для формулировки основных результатов работы нам понадобится ряд обозначений. 
Введем производящие функции чисел непосредственных потомков одной частицы первого и второго типов соответственно: для $s_{1}, s_{2} \in[0,1]$ положим

$$
f_{1}\left(s_{1}, s_{2}\right):=\mathbf{E}\left[s_{1}^{\xi_{11}} s_{2}^{\xi_{12}}\right], \quad f_{2}\left(s_{1}, s_{2}\right)=f_{2}\left(s_{2}\right):=\mathbf{E}\left[s_{2}^{\xi_{22}}\right]
$$

где $\xi_{i j}$ - число непосредственных потомков типа $j$ у частицы типа $i$.

В дальнейшем предполагается, что выполнены следующие условия:

1) $\xi_{11}=\xi_{12}$ и, таким образом, $f_{1}\left(s_{1}, s_{2}\right):=\mathbf{E}\left[\left(s_{1} s_{2}\right)^{\xi_{11}}\right]=f\left(s_{1} s_{2}\right)$ для некоторой производящей функции $f(s)$,

2 ) распределение целочисленной неотрицательной случайной величины $\xi_{11}$ имеет единичный шаг, причем

$$
\mathbf{E} \xi_{11}=1, \quad f_{1}\left(s_{1}, 1\right)=f\left(s_{1}\right)=s_{1}+\left(1-s_{1}\right)^{1+\alpha_{1}} L_{1}\left(1-s_{1}\right),
$$

$3)$ распределение целочисленной неотрицательной случайной величины $\xi_{22}$ имеет единичный шаг, причем

$$
\mathbf{E} \xi_{22}=1, \quad f_{2}(s)=s+(1-s)^{1+\alpha_{2}} L_{2}(1-s)
$$

При этом в условиях (1) и (2) параметры $\alpha_{1} \in(0,1], \alpha_{2} \in(0,1)$ удовлетворяют соотношению

$$
\frac{\alpha_{1}}{1+\alpha_{1}}<\alpha_{2}
$$

а функции $L_{i}(x), i=1,2$, медленно меняются при $x \downarrow 0$.

Пусть $\left\{\xi_{11}^{(i)}, i \geqslant 1\right\},\left\{\xi_{22}^{(i)}, i \geqslant 1\right\}$ - последовательности независимых вероятностных копий случайных величин $\xi_{11}$ и $\xi_{22}$ соответственно. Для $i=1,2$ положим

$$
S_{0}^{(i)}=0, \quad S_{n}^{(i)}:=\sum_{k=1}^{n} \xi_{i i}^{(k)}-n=\sum_{k=1}^{n}\left(\xi_{i i}^{(k)}-1\right) .
$$

Известно (см., например, [24]), что при выполнении условий (1) и (2) найдутся такие монотонно возрастающие последовательности положительных чисел

$$
a_{n}=n^{\frac{1}{1+\alpha_{1}}} l_{1}(n), b_{n}=n^{\frac{1}{1+\alpha_{2}}} l_{2}(n), n \geqslant 1,
$$

где функции $l_{i}(n), i=1,2$, медленно меняются при $n \rightarrow \infty$, что распределения случайных величин $S_{n}^{(1)} / a_{n}$ и $S_{n}^{(2)} / b_{n}$ сходятся при $n \rightarrow \infty$ к устойчивым распределениям с параметрами $1+\alpha_{1}$ и $1+\alpha_{2}$ и плотностями $g_{1}(x)$ и $g_{2}(x)$ соответственно.

Положим

$$
\begin{gathered}
R:=\int_{0}^{\infty} \frac{1}{y^{1 /\left(1+\alpha_{1}\right)}} g_{2}(-y) d y, \\
C_{1}:=\frac{1}{\left(1+\alpha_{1}\right)\left(1+\alpha_{2}\right) R}, C_{2}=C_{2}\left(\alpha_{2}\right):=\frac{1}{\left(1+\alpha_{2}\right) \Gamma\left(1-\alpha_{2}\right)},
\end{gathered}
$$

где $\Gamma(n)$ - стандартная гамма-функция, и $C_{3}:=R^{-1}$.

Пусть

$$
\left\{Y_{2}(w), w \in[0,+\infty)\right\}
$$


- ветвящийся процесс с непрерывным множеством состояний и п.н. непрерывными траекториями, преобразование Лапласа которого в момент $w \in[0, \infty)$ имеет вид

$$
\mathbf{E}\left[e^{-\lambda Y_{2}(w)}\right]=\exp \left\{-\frac{\lambda}{\left(1+w \lambda^{\alpha_{2}}\right)^{1 / \alpha_{2}}}\right\}, \quad \lambda \geqslant 0 .
$$

Введем случайную величину $\mathcal{Y}_{\infty}$, распределение которой описывается соотношением

$$
\mathbf{P}\left(\mathcal{Y}_{\infty} \leqslant x\right)=\mathbf{P}\left(\int_{0}^{\infty} Y_{2}(v) d v \leqslant x\right) .
$$

Напомним, что нормированной экскурсией Леви

$$
\mathcal{V}=\{V(s), 0 \leqslant s \leqslant 1\}
$$

с начальным условием $V(0)=0$ называется предел по распределению при $\epsilon \rightarrow 0$ устойчивого процесса Леви, рассматриваемого при условии, что его инфимум на $[0,1]$ больше $-\epsilon$, а значение при $s=1$ меньше $\epsilon$ (см. также [18] или гл. VIII.4 в монографии [20]).

С нормированной экскурсией Леви можно связать так называемый процесс кумулятивного профиля высоты $\mathcal{S}:=\{S(y), y \geqslant 0\}$, задаваемый соотношением

$$
S(y):=\sup \left\{z \leqslant 1: \int_{0}^{z} \frac{d t}{V(t)} \leqslant y\right\} \text {. }
$$

Известно [18], что процессу $\mathcal{S}$ соответствует процесс $\mathcal{U}:=\{U(t), t \geqslant 0\}$ с почти наверное непрерывными справа траекториями, который определяется равенством

$$
S(y)=\int_{0}^{y} U(t) d t, y \geqslant 0
$$

Основными результатами данной работы являются следующие две теоремы.

Теорема 1. Если выполнены условия 1)-3), то для любых $t>0$ и $x>0$

$$
\lim _{N \rightarrow \infty} \mathbf{P}\left(\frac{Z_{1}\left(\left[t b_{N} / a_{b_{N}}\right]\right)}{a_{b_{N}}} \leqslant x \mid \Xi_{2}>N\right)=C_{1} \int_{0}^{\infty} \frac{B(t ; x, y)}{y^{1+1 /\left(1+\alpha_{1}\right)}} d y,
$$

где

$$
B(t ; x, y):=\mathbf{P}\left(U\left(\frac{t}{y^{\alpha_{1} /\left(1+\alpha_{1}\right)}}\right) \leqslant \frac{x}{y^{1 /\left(1+\alpha_{1}\right)}}\right) \mathbf{P}\left(\mathcal{Y}_{\infty}>\frac{\alpha_{2}}{C_{2} y^{1+\alpha_{2}}}\right)
$$

Замечание 1. Полагая чисто формально в $(11) \quad C_{2}=C_{2}(1)=1$ и требуя конечность дисперсий случайных величин $\xi_{11}$ и $\xi_{22}$, мы получим в правой части (10) предельное распределение, совпадающее с пределом $\lim _{N \rightarrow \infty} \mathbf{P}\left(\frac{Z_{1}([t \sqrt{N}])}{\sqrt[4]{N}} \leqslant x \mid \Xi_{2}>N\right)$, найденным в работе [3]. 
Теорема 2. Если выполнены условия 1)-3), то для любых $t>0$ u $x>0$

$$
\lim _{N \rightarrow \infty} \mathbf{P}\left(\frac{Z_{1}\left(\left[t b_{N} / a_{b_{N}}\right]\right)}{a_{b_{N}}} \leqslant x \mid \Xi_{2}=N\right)=C_{3} \int_{0}^{\infty} \frac{H(t ; x, y)}{y^{1 /\left(1+\alpha_{1}\right)}} d y
$$

¿əe

$$
H(t ; x, y):=\mathbf{P}\left(U\left(\frac{t}{y^{\alpha_{1} /\left(1+\alpha_{1}\right)}}\right) \leqslant \frac{x}{y^{1 /\left(1+\alpha_{1}\right)}}\right) g_{2}(-y) .
$$

\section{2. Вспомогательные результаты}

Известно (см., например, [16]), что если выполнено условие (2), то

$$
Q_{2}(n):=\mathbf{P}\left(Z_{2}(n)>0 \mid \mathbf{Z}(0)=(0,1)\right)=\frac{1}{n^{1 / \alpha_{2}} L_{3}(n)},
$$

где функция $L_{3}(n)$ медленно меняется при $n \rightarrow \infty$.

Для $x \geqslant 1$ введем функцию

$$
G(x)=\frac{1}{Q_{2}(n)}+(x-n)\left(\frac{1}{Q_{2}(n+1)}-\frac{1}{Q_{2}(n)}\right), n \leqslant x<n+1,
$$

и $h(y)=G^{-1}(y)$ - функция, обратная к $G(x)$. Нам понадобится следующее утверждение, явно связывающее $h(n)$ с асимптотическими свойствами производящей функции $f_{2}(s)$ при $s \uparrow 1$.

Лемма 1. Если выполнено условие (2), то при $n \rightarrow \infty$

$$
h(n) \sim \frac{n^{\alpha_{2}}}{\alpha_{2} L_{2}(1 / n)} .
$$

Доказательство. Известно (см. [16]), что при $n \rightarrow \infty$

$$
\frac{1}{Q_{2}^{\alpha_{2}}(n) L_{2}\left(Q_{2}(n)\right)} \sim \alpha_{2} n
$$

Положим

$$
N=G(n)=\left[\frac{1}{Q_{2}(n)}\right]
$$

Тогда

$$
n=G^{-1}(G(n))=G^{-1}(N)=h(N) .
$$

Из (15) следует, что при $N \rightarrow \infty$

$$
\frac{N^{\alpha_{2}}}{L_{2}(1 / N)} \sim \alpha_{2} h(N) .
$$

Лемма доказана. 
Нетрудно проверить, что если выполнено условие $(2)$ и $\alpha_{2}<1$, то при $x \rightarrow \infty$

$$
\mathbf{P}\left(\xi_{22}>x\right) \sim C_{2} \frac{1}{x^{1+\alpha_{2}}} L_{2}(1 / x) \sim \frac{C_{2}}{\alpha_{2} x h(x)},
$$

где $C_{2}$ - константа из (6), а $L_{2}(x)$ - та же медленно меняющаяся при $x \downarrow 0$ функция, что и в (2). Более того, в качестве нормирующей последовательности $\left\{b_{n}, n \geqslant 1\right\}$, обеспечивающей сходимость последовательности $\left\{S_{n}^{(2)} / b_{n}, n \geqslant 1\right\}$ к устойчивому закону, можно выбрать последовательность, задаваемую равенствами

$$
b_{n}:=\min \left\{k: \mathbf{P}\left(\xi_{22}>k\right) \leqslant \frac{1}{n}\right\}, n \geqslant 1 .
$$

Из (17), учитывая соотношение (16), получаем, что при $N \rightarrow \infty$

$$
\frac{b_{N}^{1+\alpha_{2}}}{L_{2}\left(1 / b_{N}\right) C_{2}} \sim \frac{\alpha_{2} b_{N} h\left(b_{N}\right)}{C_{2}} \sim N
$$

Лемма 2. Случайная величина $\Xi_{2}$ и процесс $\left\{Z_{1}(k), k \geqslant 0\right\}$, рассматриваемые при условии $\Xi_{1}=N$, независимы.

Доказательство. Достаточно показать, что для любых значений $s_{1}, s_{2}, \ldots, s_{N}$ и $s$, принадлежащих интервалу [0,1], справедливо равенство

$$
\mathbf{E}\left[s^{\Xi_{2}} \prod_{k=1}^{N} s_{k}^{Z_{1}(k)} \mid \Xi_{1}=N\right]=\mathbf{E}\left[s^{\Xi_{2}} \mid \Xi_{1}=N\right] \mathbf{E}\left[\prod_{k=1}^{N} s_{k}^{Z_{1}(k)} \mid \Xi_{1}=N\right] .
$$

Обозначим $F(s)=\mathbf{E}\left[s^{\eta}\right], s \in[0,1]$, производящую функцию совокупного числа $\eta=Z_{2}(0)+Z_{2}(1)+\cdots$ частиц второго типа, существовавших в ветвящемся процессе за время его эволюции, в предположении, что процесс стартовал в нулевой момент времени с одной частицы второго типа. Пусть $\theta(k), k \geqslant 1,-$ число частиц второго типа в исходном ветвящемся процессе, которые являются непосредственными потомками частиц первого типа $(k-1)$-го поколения. В силу наших допущений $\theta(k)=Z_{1}(k)$ при всех $k \geqslant 1$. Для $i$-й частицы второго типа, принадлежащей $k$-му поколению $(i=1, \ldots, \theta(k))$, обозначим через $\eta_{i}(k)$ совокупное число частиц второго типа, существовавших в ветвящемся подпроцессе, порожденном именно этой частицей, до момента его вырождения. Заметим, что случайные величины $\eta_{i}(k)$, рассматриваемые при всевозможных $i, k$, независимы в совокупности и распределены так же, как и случайная величина $\eta$. Предполагая, что $z_{1}+z_{2}+\cdots+z_{N}=N-1$, введем событие

$$
A_{z_{1}, \ldots, z_{N}}:=\left\{Z_{1}(1)=z_{1}, \ldots, Z_{1}(N)=z_{N}\right\}=\left\{\theta(1)=z_{1}, \ldots, \theta(N)=z_{N}\right\} .
$$

Учитывая, что при $\Xi_{1}=N$ справедливо равенство

$$
\Xi_{2}=\sum_{k=1}^{N} \sum_{i=1}^{\theta(k)} \eta_{i}(k)
$$


несложно понять, что

$$
\begin{gathered}
\mathbf{E}\left[s^{\Xi_{2}} \mid A_{z_{1}, \ldots, z_{N}}, \Xi_{1}=N\right]=\mathbf{E}\left[\prod_{k=1}^{N} s^{\sum_{i=1}^{\theta(k)} \eta_{i}(k)} \mid A_{z_{1}, \ldots, z_{N}}, \Xi_{1}=N\right] \\
=\mathbf{E}\left[\prod_{k=1}^{N} F^{\theta(k)}(s) \mid A_{z_{1}, \ldots, z_{N}}, \Xi_{1}=N\right]=F^{N-1}(s) .
\end{gathered}
$$

Отсюда следует, что

$$
\begin{aligned}
\mathbf{E}\left[s^{\Xi_{2}}\right. & \left.\prod_{k=1}^{N} s_{k}^{Z_{1}(k)} \mid \Xi_{1}=N\right] \\
& =\mathbf{E}\left[\mathbf{E}\left[s^{\Xi_{2}} \prod_{k=1}^{N} s_{k}^{Z_{1}(k)} \mid Z_{1}(1)=z_{1}, \ldots, Z_{1}(N)=z_{N} ; \Xi_{1}=N\right]\right] \\
& =F^{N-1}(s) \mathbf{E}\left[\prod_{k=1}^{N} s_{k}^{Z_{1}(k)} \mid \Xi_{1}=N\right] .
\end{aligned}
$$

Лемма доказана.

Напомним, что согласно локальной теореме Гнеденко (см. 550 гл. IX в [10], стр. 252)

$$
\begin{aligned}
& \lim _{n \rightarrow \infty} \sup _{k}\left|a_{n} \mathbf{P}\left(S_{n}^{(1)}=k\right)-g_{1}\left(\frac{k}{a_{n}}\right)\right|=0 \\
& \lim _{n \rightarrow \infty} \sup _{k}\left|b_{n} \mathbf{P}\left(S_{n}^{(2)}=k\right)-g_{2}\left(\frac{k}{b_{n}}\right)\right|=0 .
\end{aligned}
$$

Нам понадобится следующий результат, являющийся переформулировкой теоремы 1 работы [6] применительно к нашим условиям.

Лемма 3 ([6]). Если выполнены условия 1)-3), то при $N \rightarrow \infty$

$$
\mathcal{L}\left(\frac{\Xi_{2}([w h(N)])}{N h(N)}, 0 \leqslant w<\infty \mid \Xi_{1}=N\right) \Longrightarrow \mathcal{L}\left(\int_{0}^{w} Y_{2}(v) d v, 0 \leqslant w<\infty\right),
$$

где символ $\Longrightarrow$ означает слабую сходимость в функииональном пространстве $D[0,+\infty)$, оснащенном топологией Скорохода.

Для $t \geqslant 0$ положим

$$
U^{(n)}(t):=\frac{Z_{1}\left(\left[n t / a_{n}\right]\right)}{a_{n}} .
$$

Мы дополним лемму 3 важным утверждением, заимствованным из [18].

Лемма 4 ([18], теорема 1). Если распределение случайной величины $\xi_{11}$ является арифметическим с единичным шагом и выполнено условие (1), то при $N \rightarrow \infty$

$$
\mathcal{L}\left(U^{(n)}(t), t \geqslant 0 \mid \Xi_{1}=N\right) \Longrightarrow \mathcal{L}(U(t), t \geqslant 0),
$$

где прочесс $\{U(t), t \geqslant 0\}$ задан соотношением (9). 
Следующая лемма является ключевой для дальнейших выкладок. При ее доказательстве мы будем обозначать через $K_{1}, K_{2}, \ldots$ положительные константы, не обязательно совпадающие в разных формулах.

Лемма 5. Если выполнены условия 1)-3), то при $N \rightarrow \infty$

$$
\begin{gathered}
\mathbf{P}\left(\Xi_{2}=N\right) \sim g_{1}(0) \frac{R}{N a_{b_{N}}}, \\
\mathbf{P}\left(\Xi_{2}>N\right) \sim g_{1}(0) \frac{\left(1+\alpha_{1}\right)\left(1+\alpha_{2}\right) R}{a_{b_{N}}},
\end{gathered}
$$

где величина $R$ та же, что и в (5).

Доказательство. Для $j=1,2$ положим

$$
\zeta_{i j}=\xi_{j j}^{(i)}-1, i=1,2, \ldots
$$

Заметим, что $\mathbf{E} \zeta_{i j}=0$. Из теоремы Гнеденко (21) следует, что при $N \rightarrow \infty$

$$
\mathbf{P}\left(\zeta_{11}+\cdots+\zeta_{N 1}=-T\right)=\frac{1}{a_{N}} g_{1}\left(\frac{-T}{a_{N}}\right)+o\left(\frac{1}{a_{N}}\right) .
$$

Согласно лемме $3 \S 1$ гл. II в [19]

$$
\begin{aligned}
\mathbf{P}\left(\Xi_{1}=N\right) & =\frac{1}{N} \mathbf{P}\left(\xi_{11}^{(1)}+\cdots+\xi_{11}^{(N)}=N-1\right) \\
& =\frac{1}{N} \mathbf{P}\left(\zeta_{11}+\cdots+\zeta_{N 1}=-1\right) .
\end{aligned}
$$

Таким образом, при $N \rightarrow \infty$

$$
\mathbf{P}\left(\Xi_{1}=N\right) \sim \frac{1}{N} g_{1}\left(\frac{-1}{a_{N}}\right) \frac{1}{a_{N}} \sim \frac{1}{N a_{N}} g_{1}(0) .
$$

Исследуем теперь асимптотическое поведение вероятности события $\left\{\Xi_{2}=N\right\}$ при $N \rightarrow \infty$. Зафиксируем числа $C>\varepsilon>0$ и запишем представление

$$
\mathbf{P}\left(\Xi_{2}=N\right)=P_{1}(N ; \varepsilon)+P_{2}(N ; \varepsilon, C)+P_{3}(N ; C),
$$

где

$$
\begin{aligned}
P_{1}(N ; \varepsilon) & :=\mathbf{P}\left(\Xi_{2}=N, \Xi_{1}<\varepsilon b_{N}\right), \\
P_{2}(N ; \varepsilon, C) & :=\sum_{M=\varepsilon b_{N}}^{C b_{N}} \mathbf{P}\left(\Xi_{2}=N, \Xi_{1}=M\right), \\
P_{3}(N ; C) & :=\mathbf{P}\left(\Xi_{2}=N, \Xi_{1}>C b_{N}\right) .
\end{aligned}
$$

(Здесь и далее величины типа $\varepsilon b_{N}$ и $C b_{N}$ интерпретируются как $\left[\varepsilon b_{N}\right]$ и $\left[C b_{N}\right]$. )

Оценим сначала первое слагаемое

$$
P_{1}(N ; \varepsilon)=\sum_{M<\varepsilon b_{N}} \mathbf{P}\left(\Xi_{2}=N \mid \Xi_{1}=M\right) \mathbf{P}\left(\Xi_{1}=M\right) .
$$


Обозначим $\Xi_{12}$ число частиц второго типа, появившихся в процессе до момента его вырождения и являющихся потомками первого типа. В силу наших условий $\Xi_{12}=\Xi_{1}$. Ясно, что $\Xi_{2}$ является совокупным числом частиц второго типа, существовавших в ветвящемся процессе, порожденным $\Xi_{12}$ первоначальными частицами второго типа. Согласно упоминавшейся лемме 3 §1 гл. II в [19]

$$
\begin{aligned}
\mathbf{P}\left(\Xi_{2}=N \mid \Xi_{12}=M\right) & =\frac{M}{N} \mathbf{P}\left(\xi_{22}^{(1)}+\cdots+\xi_{22}^{(N)}=N-M\right) \\
& =\frac{M}{N} \mathbf{P}\left(\zeta_{12}+\cdots+\zeta_{N 2}=-M\right) .
\end{aligned}
$$

Из теоремы Гнеденко (22) следует, что при $N \rightarrow \infty$

$$
\mathbf{P}\left(\zeta_{12}+\cdots+\zeta_{N 2}=-M\right)=\frac{1}{b_{N}} g_{2}\left(\frac{-M}{b_{N}}\right)+o\left(\frac{1}{b_{N}}\right) .
$$

Это, в частности, означает, что существует такая константа $K_{1}$, что при всех $M$ и $N$

$$
\mathbf{P}\left(\Xi_{2}=N \mid \Xi_{1}=M\right) \leqslant M \frac{K_{1}}{N b_{N}} .
$$

Соотношения $(25)$ и (28) и свойства правильно меняющихся функций приводят к оценке

$$
\begin{aligned}
P_{1}(N ; \varepsilon) & \leqslant K_{2} \frac{1}{N b_{N}} \sum_{M \leqslant \varepsilon b_{N}} \frac{1}{a_{M}} \sim K_{3} \frac{1}{N b_{N}} \frac{\varepsilon b_{N}}{a_{\varepsilon b_{N}}} \\
& \sim K_{3} \frac{\varepsilon}{N a_{b_{N}} \varepsilon^{1 /\left(1+\alpha_{1}\right)}}=K_{3} \frac{\varepsilon^{\alpha_{1} /\left(1+\alpha_{1}\right)}}{N a_{b_{N}}} .
\end{aligned}
$$

Отсюда следует, что

$$
\lim _{\varepsilon \rightarrow 0} \limsup _{N \rightarrow \infty} N a_{b_{N}} P_{1}(N ; \varepsilon) \leqslant K_{3} \lim _{\varepsilon \rightarrow 0} \varepsilon^{\alpha_{1} /\left(1+\alpha_{1}\right)}=0 .
$$

Оценим теперь величину $P_{3}(N ; C)$. В силу $(25)$ и монотонного возрастания элементов последовательности $\left\{a_{n}, n \geqslant 1\right\}$ имеем

$$
\begin{aligned}
P_{3}(N ; C) & =\frac{1}{N} \sum_{M>C b_{N}} \mathbf{P}\left(\zeta_{12}+\cdots+\zeta_{N 2}=-M\right) \mathbf{P}\left(\zeta_{11}+\cdots+\zeta_{M 1}=-1\right) \\
& \leqslant \frac{K_{1}}{N} \sum_{M>C b_{N}} \frac{\mathbf{P}\left(\zeta_{12}+\cdots+\zeta_{N 2}=-M\right)}{a_{M}} \\
& \leqslant \frac{K_{1}}{N a_{C b_{N}}} \mathbf{P}\left(\zeta_{12}+\cdots+\zeta_{N 2} \geqslant C b_{N}\right) \leqslant \frac{K_{1}}{N a_{C b_{N}}}
\end{aligned}
$$

Отсюда, из (4) и свойств правильно меняющихся функций следует, что

$$
\limsup _{N \rightarrow \infty} N a_{b_{N}} P_{3}(N ; C) \leqslant K_{1} \lim _{N \rightarrow \infty} \frac{a_{b_{N}}}{a_{C b_{N}}}=K_{1} C^{-1 /\left(1+\alpha_{1}\right)}
$$

и, таким образом,

$$
\lim _{C \rightarrow \infty} \limsup _{N \rightarrow \infty} N a_{b_{N}} P_{3}(N ; C)=0 .
$$


Для завершения доказательства леммы осталось найти асимптотическое представление для величины

$$
P_{2}(N ; \varepsilon, C)=\sum_{M=\varepsilon b_{N}}^{C b_{N}} \mathbf{P}\left(\Xi_{2}=N \mid \Xi_{1}=M\right) \mathbf{P}\left(\Xi_{1}=M\right) .
$$

Обращаясь к соотношению (22), заключаем, что

$$
\begin{aligned}
\mathbf{P}\left(\Xi_{2}=\right. & \left.N \mid \Xi_{1}=M\right)=\mathbf{P}\left(\Xi_{2}=N \mid \Xi_{12}=M\right) \\
& =\frac{M}{N} \mathbf{P}\left(S_{N}^{(2)}=-M\right)=\frac{M}{N b_{N}}\left(g_{2}\left(-\frac{M}{b_{N}}\right)+\delta(M, N)\right),
\end{aligned}
$$

где $\delta(M, N) \rightarrow 0$ при $N \rightarrow \infty$ равномерно по $M \in\left[\varepsilon b_{N}, C b_{N}\right]$.

Из равенства (33), учитывая соотношение (25), получаем, что при $N \rightarrow \infty$

$$
\begin{aligned}
P_{2}(N ; \varepsilon, C) & \sim \sum_{M=\varepsilon b_{N}}^{C b_{N}} \frac{1}{M} g_{1}(0) \frac{1}{a_{M}} \frac{M}{N} g_{2}\left(-\frac{M}{b_{N}}\right) \frac{1}{b_{N}} \\
& =g_{1}(0) \frac{1}{N} \sum_{M=\varepsilon b_{N}}^{C b_{N}} \frac{1}{a_{M}} g_{2}\left(-\frac{M}{b_{N}}\right) \frac{1}{b_{N}} .
\end{aligned}
$$

В силу (4) и свойств правильно меняющихся функций

$$
a_{b_{N}} / a_{y b_{N}} \rightarrow y^{-1 /\left(1+\alpha_{1}\right)}
$$

при $N \rightarrow \infty$ равномерно по $y \in[\varepsilon, C]$. Отсюда, сделав замену $M=\left[y b_{N}\right]$ и воспользовавшись непрерывностью функции $g_{2}(y), y \in(-\infty, \infty)$, нетрудно вывести, что

$$
\lim _{N \rightarrow \infty} a_{b_{N}} \sum_{M=\varepsilon b_{N}}^{C b_{N}} \frac{1}{a_{M}} g_{2}\left(-\frac{M}{b_{N}}\right) \frac{1}{b_{N}}=\int_{\varepsilon}^{C} \frac{1}{y^{1 /\left(1+\alpha_{1}\right)}} g_{2}(-y) d y .
$$

Следовательно,

$$
\lim _{N \rightarrow \infty} N a_{b_{N}} P_{2}(N ; \varepsilon, C)=\int_{\varepsilon}^{C} \frac{1}{y^{1 /\left(1+\alpha_{1}\right)}} g_{2}(-y) d y .
$$

Так как $g_{2}(y)$ является плотностью распределения устойчивого закона, то (см., например, [22]) существует такая константа $K_{2}$, что

$$
\sup _{y \in(-\infty, \infty)} g_{2}(y) \leqslant K_{2} .
$$

Таким образом,

$$
\int_{0}^{\infty} \frac{1}{y^{1 /\left(1+\alpha_{1}\right)}} g_{2}(-y) d y \leqslant K_{2} \int_{0}^{1} \frac{1}{y^{1 /\left(1+\alpha_{1}\right)}} d y+\int_{1}^{\infty} g_{2}(-y) d y<\infty .
$$


Объединяя $(26),(30),(32)$ и (34) и переходя к пределу сначала при $C \rightarrow \infty$, а затем при $\varepsilon \rightarrow 0$, заключаем, что при $N \rightarrow \infty$

$$
\mathbf{P}\left(\Xi_{2}=N\right) \sim \frac{g_{1}(0)}{N} \frac{1}{a_{b_{N}}} \int_{0}^{\infty} \frac{1}{y^{1 /\left(1+\alpha_{1}\right)}} g_{2}(-y) d y .
$$

Справедливость (23) доказана. В силу соотношений (4) и свойств медленно меняющихся функций из (23) следует (24). Лемма доказана.

Замечание 2. Из (29), (31) и свойств правильно меняющихся функций нетрудно вывести, что

$$
\begin{aligned}
\mathbf{P}\left(\Xi_{2}>N, \Xi_{1}<\varepsilon b_{N}\right) & \leqslant K_{1} \frac{\varepsilon^{\alpha_{1} /\left(1+\alpha_{1}\right)}}{a_{b_{N}}} \\
\mathbf{P}\left(\Xi_{2}>N, \Xi_{1}>C b_{N}\right) & \leqslant \frac{K_{2}}{a_{C b_{N}}}
\end{aligned}
$$

что в сочетании с (24) позволяет утверждать, что

$$
\lim _{C \rightarrow \infty} \lim _{\varepsilon \rightarrow 0} \limsup _{N \rightarrow \infty} \frac{\mathbf{P}\left(\Xi_{2}>N, \Xi_{1} \notin\left[\varepsilon b_{N}, C b_{N}\right]\right)}{\mathbf{P}\left(\Xi_{2}>N\right)}=0 .
$$

\section{3. Доказательства теорем}

Доказательство теоремы 1. Зафиксируем числа $x>0$ и $C>\varepsilon>0$ и воспользуемся представлением

$$
\mathbf{P}\left(\frac{Z_{1}\left(\left[t b_{N} / a_{b_{N}}\right]\right)}{a_{b_{N}}} \leqslant x, \Xi_{2}>N\right)=P_{1}(N ; \varepsilon, C)+P_{2}(N ; \varepsilon, C),
$$

где

$$
\begin{aligned}
& P_{1}(N ; \varepsilon, C):=\mathbf{P}\left(\frac{Z_{1}\left(\left[t b_{N} / a_{b_{N}}\right]\right)}{a_{b_{N}}} \leqslant x, \Xi_{2}>N, \Xi_{1} \notin\left[\varepsilon b_{N}, C b_{N}\right]\right), \\
& P_{2}(N ; \varepsilon, C):=\mathbf{P}\left(\frac{Z_{1}\left(\left[t b_{N} / a_{b_{N}}\right]\right)}{a_{b_{N}}} \leqslant x, \Xi_{2}>N, \Xi_{1} \in\left[\varepsilon b_{N}, C b_{N}\right]\right) .
\end{aligned}
$$

В силу (35)

$$
\lim _{\varepsilon \rightarrow 0} \lim _{C \rightarrow \infty} \limsup _{N \rightarrow \infty} \frac{P_{1}(N ; \varepsilon, C)}{\mathbf{P}\left(\Xi_{2}>N\right)}=0,
$$

в связи с чем интерес представляет лишь второе слагаемое в правой части (36), которое, ввиду леммы 2 , можно записать в виде

$$
\begin{gathered}
\sum_{M=\varepsilon b_{N}}^{C b_{N}} \mathbf{P}\left(\frac{Z_{1}\left(\left[t b_{N} / a_{b_{N}}\right]\right)}{a_{b_{N}}} \leqslant x, \Xi_{2}>N \mid \Xi_{1}=M\right) \mathbf{P}\left(\Xi_{1}=M\right) \\
=\sum_{M=\varepsilon b_{N}}^{C b_{N}} \mathbf{P}\left(\frac{Z_{1}\left(\left[t b_{N} / a_{b_{N}}\right]\right)}{a_{b_{N}}} \leqslant x \mid \Xi_{1}=M\right) \\
\times \mathbf{P}\left(\Xi_{2}>N \mid \Xi_{1}=M\right) \mathbf{P}\left(\Xi_{1}=M\right) .
\end{gathered}
$$


Зафиксируем число $y \in[\varepsilon, C]$ и положим $M=\left[y b_{N}\right]$. Из леммы 4 и соотношений в (4) после несложных преобразований следует, что

$$
\begin{aligned}
\lim _{N \rightarrow \infty} & \mathbf{P}\left(\frac{Z_{1}\left(\left[t b_{N} / a_{b_{N}}\right]\right)}{a_{b_{N}}} \leqslant x \mid \Xi_{1}=\left[y b_{N}\right]\right) \\
& =\lim _{M \rightarrow \infty} \mathbf{P}\left(\frac{Z_{1}\left(\left[t \frac{M}{y} \frac{y^{1 /\left(1+\alpha_{1}\right)}}{a_{M}}\right]\right)}{a_{M} y^{-1 /\left(1+\alpha_{1}\right)}} \leqslant x \mid \Xi_{1}=M\right) \\
& =\mathbf{P}\left(U\left(\frac{t}{y^{\alpha_{1} /\left(1+\alpha_{1}\right)}}\right) \leqslant \frac{x}{y^{1 /\left(1+\alpha_{1}\right)}}\right)
\end{aligned}
$$

для всех $x$ и $y$, для которых правая часть в (38) непрерывна.

Найдем теперь для $M=\left[y b_{N}\right]$ асимптотику условной вероятности

$$
\mathbf{P}\left(\Xi_{2}>N \mid \Xi_{1}=M\right)
$$

при $N \rightarrow \infty$.

Из леммы 3 вытекает, что

$$
\lim _{M \rightarrow \infty} \mathbf{P}\left(\frac{\Xi_{2}}{M h(M)} \geqslant x \mid \Xi_{1}=M\right)=\mathbf{P}\left(\mathcal{Y}_{\infty} \geqslant x\right) .
$$

Отсюда и из (18) следует, что для $y \in[\varepsilon, C]$ и $M=\left[y b_{N}\right]$

$$
\mathbf{P}\left(\Xi_{2}>N \mid \Xi_{1}=M\right)=\mathbf{P}\left(\Xi_{2}>\frac{\alpha_{2} b_{N} h\left(b_{N}\right)(1+o(1))}{C_{2}} \mid \Xi_{1}=M\right) .
$$

Из соотношения (39) и лемм 1 и 3 нетрудно вывести, что если $M=\left[y b_{N}\right]$ и $y \in[\varepsilon, C]$, то при $N \rightarrow \infty$

$$
\mathbf{P}\left(\frac{\Xi_{2}}{M h(M)}>\frac{\alpha_{2}}{y_{2}^{1+\alpha_{2}} C_{2}} \mid \Xi_{1}=M\right) \sim \mathbf{P}\left(\mathcal{Y}_{\infty}>\frac{\alpha_{2}}{C_{2} y^{1+\alpha_{2}}}\right)
$$

для всех $y$, для которых правая часть в (40) непрерывна. Следовательно, для почти всех $y \in[\varepsilon, C]$

$$
\lim _{N \rightarrow \infty} \mathbf{P}\left(\Xi_{2}>N \mid \Xi_{1}=M\right)=\mathbf{P}\left(\mathcal{Y}_{\infty}>\frac{\alpha_{2}}{C_{2} y^{1+\alpha_{2}}}\right)
$$

Положим

$$
\Phi(M, N):=\frac{\mathbf{P}\left(\Xi_{1}=M\right)}{\mathbf{P}\left(\Xi_{2}>N\right)} .
$$

Заметим теперь, что из соотношений $(24),(25)$ и свойств правильно меняющихся функций следует, что при $N \rightarrow \infty$

$$
\Phi(M, N) \sim \frac{C_{1}}{b_{N} y^{1+1 /\left(1+\alpha_{1}\right)}}=: \frac{\phi(y)}{b_{N}}
$$


равномерно по $M=\left[y b_{N}\right], y \in[\varepsilon, C]$, где $C_{1}$ - та же константа, что и в (6). Объединяя (38), (41) и (42) и используя теорему о мажорируемой сходимости, заключаем, что при $N \rightarrow \infty$

$$
\begin{aligned}
& \sum_{M=\varepsilon b_{N}}^{C b_{N}} \mathbf{P}\left(\frac{Z_{1}\left(\left[t b_{N} / a_{b_{N}}\right]\right)}{a_{b_{N}}} \leqslant x, \Xi_{2}>N, \Xi_{1}=M\right) \frac{1}{\mathbf{P}\left(\Xi_{2}>N\right)} \\
\sim & \int_{\varepsilon}^{C} \mathbf{P}\left(\frac{Z_{1}\left(\left[t b_{N} / a_{b_{N}}\right]\right)}{a_{b_{N}}} \leqslant x \mid \Xi_{1}=\left[y b_{N}\right]\right) \mathbf{P}\left(\Xi_{2}>N \mid \Xi_{1}=\left[y b_{N}\right]\right) \Phi\left(\left[y b_{N}\right], N\right) d y \\
\sim & \int_{\varepsilon}^{C} \mathbf{P}\left(U\left(\frac{t}{y^{\alpha_{1} /\left(1+\alpha_{1}\right)}}\right) \leqslant \frac{x}{y^{1 /\left(1+\alpha_{1}\right)}}\right) \mathbf{P}\left(\mathcal{Y}_{\infty}>\frac{\alpha_{2}}{C_{2} y^{1+\alpha_{2}}}\right) \phi(y) d y .
\end{aligned}
$$

Из соотношений $(36),(37)$ и (43), переходя в последнем из них к пределу сначала при $C \rightarrow \infty$, а потом при $\varepsilon \rightarrow 0$, получаем

$$
\lim _{N \rightarrow \infty} \mathbf{P}\left(\frac{Z_{1}\left(\left[t b_{N} / a_{b_{N}}\right]\right)}{a_{b_{N}}} \leqslant x, \Xi_{2}>N\right) \frac{1}{\mathbf{P}\left(\Xi_{2}>N\right)}=C_{1} \int_{0}^{\infty} \frac{B(t ; x, y)}{y^{1+1 /\left(1+\alpha_{1}\right)}} d y
$$

Теорема 1 доказана.

Доказательство теоремы 2. Аналогично доказательству предыдущей теоремы, для фиксированных чисел $x>0$ и $C>\varepsilon>0$ имеем

$$
\mathbf{P}\left(\frac{Z_{1}\left(\left[t b_{N} / a_{b_{N}}\right]\right)}{a_{b_{N}}} \leqslant x, \Xi_{2}=N\right)=\mathcal{P}_{1}(N ; \varepsilon, C)+\mathcal{P}_{2}(N ; \varepsilon, C),
$$

где

$$
\begin{aligned}
& \mathcal{P}_{1}(N ; \varepsilon, C):=\mathbf{P}\left(\frac{Z_{1}\left(\left[t b_{N} / a_{b_{N}}\right]\right)}{a_{b_{N}}} \leqslant x, \Xi_{2}=N, \Xi_{1} \notin\left[\varepsilon b_{N}, C b_{N}\right]\right), \\
& \mathcal{P}_{2}(N ; \varepsilon, C):=\mathbf{P}\left(\frac{Z_{1}\left(\left[t b_{N} / a_{b_{N}}\right]\right)}{a_{b_{N}}} \leqslant x, \Xi_{2}=N, \Xi_{1} \in\left[\varepsilon b_{N}, C b_{N}\right]\right) .
\end{aligned}
$$

В силу (30) и (32)

$$
\lim _{C \rightarrow \infty} \lim _{\varepsilon \rightarrow 0} \limsup _{N \rightarrow \infty} \frac{\mathcal{P}_{1}(N ; \varepsilon, C)}{\mathbf{P}\left(\Xi_{2}=N\right)}=0 .
$$

Рассмотрим теперь величину

$$
\begin{aligned}
& \mathcal{P}_{2}(N ; \varepsilon, C) \\
& =\sum_{M=\varepsilon b_{N}}^{C b_{N}} \mathbf{P}\left(\frac{Z_{1}\left(\left[t b_{N} / a_{b_{N}}\right]\right)}{a_{b_{N}}} \leqslant x, \Xi_{2}=N \mid \Xi_{1}=M\right) \mathbf{P}\left(\Xi_{1}=M\right) \\
& =\sum_{M=\varepsilon b_{N}}^{C b_{N}} \mathbf{P}\left(\frac{Z_{1}\left(\left[t b_{N} / a_{b_{N}}\right]\right)}{a_{b_{N}}} \leqslant x \mid \Xi_{1}=M\right) \times \mathbf{P}\left(\Xi_{2}=N \mid \Xi_{1}=M\right) \mathbf{P}\left(\Xi_{1}=M\right),
\end{aligned}
$$


где для оправдания знака равенства мы воспользовались леммой 2 .

Из теоремы Гнеденко (22), учитывая свойства правильно меняющихся функций, получаем, что при $N \rightarrow \infty$

$$
\mathbf{P}\left(\Xi_{2}=N \mid \Xi_{1}=\left[y b_{N}\right]\right) \sim \frac{y b_{N}}{N b_{N}} g_{2}\left(\frac{-y b_{N}}{b_{N}}\right) \sim \frac{y}{N} g_{2}(-y) .
$$

равномерно по $y \in[\varepsilon, C]$. Кроме того, в силу асимптотического представления (25) при $N \rightarrow \infty$

$$
\mathbf{P}\left(\Xi_{1}=M\right) \sim \frac{1}{y b_{N}} g_{1}(0) \frac{1}{y^{1 /\left(1+\alpha_{1}\right)} a_{b_{N}}} \sim \frac{g_{1}(0)}{y^{1+1 /\left(1+\alpha_{1}\right)}} \frac{1}{b_{N} a_{b_{N}}}
$$

равномерно по $M=\left[y b_{N}\right], y \in[\varepsilon, C]$.

Отсюда и из леммы 5 следует, что при $N \rightarrow \infty$

$$
\begin{aligned}
\frac{\mathbf{P}\left(\Xi_{1}=M\right)}{\mathbf{P}\left(\Xi_{2}=N\right)} & \sim \frac{g_{1}(0)}{y^{1+1 /\left(1+\alpha_{1}\right)}} \frac{1}{b_{N} a_{b_{N}}} \frac{N a_{b_{N}}}{g_{1}(0)} \frac{1}{R} \\
& \sim \frac{1}{y^{1+1 /\left(1+\alpha_{1}\right)}} \frac{N}{b_{N}} \frac{1}{R}
\end{aligned}
$$

равномерно по $M=\left[y b_{N}\right], y \in[\varepsilon, C]$. Объединяя соотношения $(38),(46)$ и (47) и обращаясь к теореме о мажорируемой сходимости, выводим, что при $N \rightarrow \infty$

$$
\begin{gathered}
\sum_{M=\varepsilon b_{N}}^{C b_{N}} \mathbf{P}\left(\frac{Z_{1}\left(\left[t b_{N} / a_{b_{N}}\right]\right)}{a_{b_{N}}} \leqslant x, \Xi_{2}=N, \Xi_{1}=M\right) \frac{1}{\mathbf{P}\left(\Xi_{2}=N\right)} \\
\sim \int_{\varepsilon b_{N}}^{C b_{N}} \mathbf{P}\left(\frac{Z_{1}\left(\left[t b_{N} / a_{b_{N}}\right]\right)}{a_{b_{N}}} \leqslant x, \Xi_{2}=N, \Xi_{1}=\left[y b_{N}\right]\right) \frac{d y}{\mathbf{P}\left(\Xi_{2}=N\right)} \\
\sim C_{3} \int_{\varepsilon}^{C} \mathbf{P}\left(U\left(\frac{t}{y^{\alpha_{1} /\left(1+\alpha_{1}\right)}}\right) \leqslant \frac{x}{y^{1 /\left(1+\alpha_{1}\right)}}\right) \frac{g_{2}(-y)}{y^{1 /\left(1+\alpha_{1}\right)}} d y .
\end{gathered}
$$

Из соотношений (44), (45) и (48), переходя в последнем к пределу сначала при $C \rightarrow \infty$, а потом при $\varepsilon \rightarrow 0$, нетрудно заключить, что

$$
\lim _{N \rightarrow \infty} \mathbf{P}\left(\frac{Z_{1}\left(\left[t b_{N} / a_{b_{N}}\right]\right)}{a_{b_{N}}} \leqslant x, \Xi_{2}=N\right) \frac{1}{\mathbf{P}\left(\Xi_{2}=N\right)}=C_{3} \int_{0}^{\infty} \frac{H(t ; x, y)}{y^{1 /\left(1+\alpha_{1}\right)}} d y
$$

Теорема 2 доказана.

Авторы выражают благодарность рецензенту, замечания которого способствовали улучшению изложения полученных результатов. 


\section{Список литературы}

1. Афанасьев В. И., "Функциональные предельные теоремы для разложимого ветвящегося процесса с двумя типами частиц", Дискретная математика, 27:2 (2015), 22-44; англ. пер.: Afanasyev V. I., "Functional limit theorems for the decomposable branching process with two types of particles", Discrete Math. Appl., 26:2 (2016), 71-88.

2. Афанасьев В. И., "О разложимом ветвящемся процессе с двумя типами частиц", Современные проблемы математики, механики и математической физики. II, Сборник статей, Тр. МИАН, МАИК Москва, 294 (2016), 7-19; англ. пер.: Afanasyev V. I., "On a decomposable branching process with two types of particles", Proc. Steklov Inst. Math., 294 (2016), 1-12.

3. Афанасьев В. И., "Функциональная предельная теорема для разложимого ветвящегося процесса с двумя типами частиц", Матем. заметки, 103:3 (2018), 323-335; англ. пер.: Afanasyev V. I., "Functional limit theorem for a decomposable branching process with two types of particles", in print, Math. Notes, 103:3 (2018).

4. Ватутин В. А., "Структура разложимых редуцированных ветвящихся процессов. I. Конечномерные распределения", Теория вероятн. и ее применен., 59:4 (2014), 667-692; англ. пер.: Vatutin V. A., "The structure of decomposable reduced branching processes. I. Finite-dimensional distributions", Theory Probab. Appl., 59:4 (2015), 641-662.

5. Ватутин В. А., "Структура разложимых редуцированных ветвящихся процессов. II. Функциональные предельные теоремы”, Теория вероятн. и ее применен., 60:1 (2015), 25-44; англ. пер.: Vatutin V. A., "The structure of decomposable reduced branching processes. II. Functional limit theorems", Theory Probab. Appl., 60:1 (2016), 103-119.

6. Ватутин В. А., "Условная функциональная предельная теорема для разложимых ветвящихся процессов с двумя типами частиц", Матем. заметки, 101:5 (2017), 669-683; англ. пер.: Vatutin V. A., "A conditional functional limit theorem for decomposable branching processes with two types of particles", Math. Notes, 101:5 (2017), 778-789.

7. Ватутин В. А., Дьяконова Е. Е., "Разложимые ветвящиеся процессы с фиксированным моментом вырождения", Современные проблемы математики, механики и математической физики, Сборник статей, Тр. МИАН, МАИК, Москва, 290 (2015), 114-135; англ. пер.: Vatutin V. A., D'yakonova E. E., "Decomposable branching processes with a fixed extinction moment", Proc. Steklov Inst. Math., 290 (2015), 103-124.

8. Ватутин В. А., Дьяконова Е. Е., "О вырождении разложимых ветвящихся процессов", Дискретная математика, 28:4 (2015), 26-37; англ. пер.: Vatutin V. A., Dyakonova E. E., "Extinction of decomposable branching processes", Discrete Math. Appl., 26:3 (2016), 183-192.

9. Ватутин В. А. , Сагитов С. М., "Разложимый критический ветвящийся процесс с двумя типами частиц", Вероятностные задачи дискретной математики, Тр. МИАН СССР, 177 (1986), 3-20; англ. пер.: Vatutin V. A., Sagitov S. M., "A decomposable critical branching process with two types of particles", Proc. Steklov Inst. Math., 177 (1988), 1-19.

10. Гнеденко Б. В., Колмогоров А. Н., Предельные теоремы для сумм независимых случайных величин, Гос. из-во технико-теоретической литературы, Москва, Ленинград, 1949; англ. пер.: Gnedenko B. V., Kolmogorov A. N., Limit distributions for sums of independent random variables, Addison-Wesley, Reading, London, 1954.

11. Foster J., Ney P., "Decomposable critical multi-type branching processes", Invited paper for Mahalanobis Memorial symposium (Calcutta), Sanhya: the Indian J. Stat., Series A, 38 (1976), 28-37.

12. Foster J., Ney P., "Limit laws for decomposable critical branching processes", $Z$. Wahrscheinlichkeitstheorie verw. Gebiete, 46 (1978), 13-43.

13. Ogura Y., "Asymptotic behavior of multitype Galton-Watson processes", J. Math. Kyoto Univ., 15 (1975), 251-302. 
14. Савин А. А., Чистяков В. П., "Некоторые теоремы для ветвящихся процессов с несколькими типами частиц", Теория вероятн. и ее применен., 7:1 (1962), 95-104; англ. пер.: Savin A.A., Chistyakov V.P., "Some limit theorems for branching processes with several types of particles", Theory Probab. Appl., 7:1 (1962), 93-100.

15. Сагитов С. М., "Многомерные предельные теоремы для ветвящегося процесса с одним типом частиц", Матем. заметки, 42:1 (1987), 157-165; англ. пер.: Sagitov S. M.,, "Multidimensional limit theorems for a branching process with a single type of particles", Math. Notes, 42:1 (1987), 597-602.

16. Slack R. S., "A branching process with mean one and possibly infinite variance", $Z$. Wahrscheinlichkeitstheorie verw. Gebiete, 9:2 (1968), 139-145.

17. Сенета Е., Правильно меняющиеся функции, Наука, Москва, 1985; пер. с англ.: Seneta E., Regularly Varying Functions, Springer-Verlag, Berlin, Heidelberg, New York, 1976.

18. Kersting G., "On the height profile of a conditioned Galton-Watson tree", arXiv:1101.3656 [math.PR], 2011, 24 pages.

19. Колчин В. Ф., Случайные отображения, Наука, Москва, 1984; англ. пер.: Kolchin V. F., Random Mappings, Optimization Software, Inc., Publication Division, 1986.

20. Bertoin J., Levy Processes, Cambrige University Press, Cambridge, 1996.

21. Smadi C., Vatutin V. A., "Reduced two-type decomposable critical branching processes with possibly infinite variance", Markov Process. Related Fields, 22:2 (2016), 311-358.

22. Золотарев В. М., Одномерные устойчивые распределения, Наука, Москва, 1983; англ. пер.: Zolotarev V. M., One-dimensional Stable Distributions, Transl. of Math. Monographs, vol. 65, Amer. Math. Soc., Providence, 1986.

23. Зубков А. М., "Предельное поведение разложимых критических ветвящихся процессов с двумя типами частиц", Теория вероятн. и ее применен., 27:2 (1982), 228-238; англ. пер.: Zubkov A. M., "The limit behaviour of decomposable critical branching processes with two types of particles", Theory Probab. Appl., 27:2 (1983), 235-237.

24. Феллер В., Введение в теорию вероятностей и ее приложения, m. 2, Мир, Москва, 1984; англ. пер.: Feller W., An Introduction to Probability Theory and its Applications. Vol II, John Wiley\& Sons, Inc., New York, London, Sydney, Toronto, 1971. 